PREPARED FOR THE U.S. DEPARTMENT OF ENERGY, UNDER CONTRACT DE-AC02-76CH03073

PPPL-3734

PPPL-3734

UC-70

Evaluation of Possible Nuclear Magnetic Resonance Diagnostic Techniques for Tokamak Experiments

by

S.J. Zweben, T.W. Kornack, D.Majeski, G. Schilling, C.H. Skinner, R. Wilson, and N. Kuzma

August 2002

N/

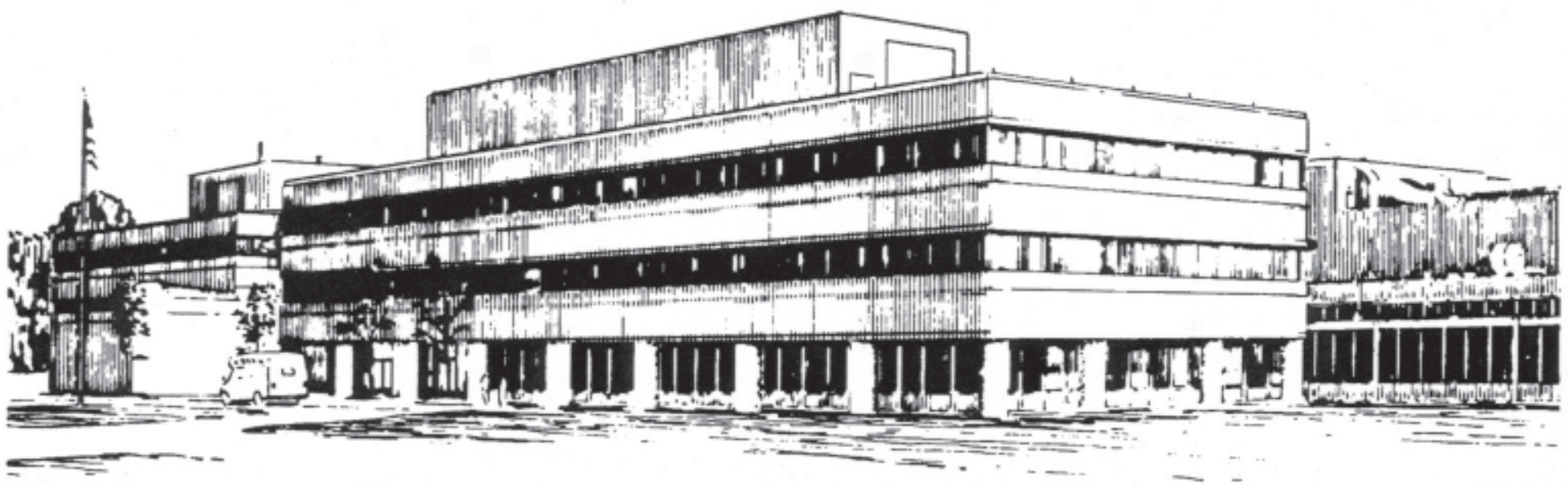

PRINCETON PLASMA PHYSICS LABORATORY PRINCETON UNIVERSITY, PRINCETON, NEW JERSEY 


\section{PPPL Reports Disclaimer}

This report was prepared as an account of work sponsored by an agency of the United States Government. Neither the United States Government nor any agency thereof, nor any of their employees, makes any warranty, express or implied, or assumes any legal liability or responsibility for the accuracy, completeness, or usefulness of any information, apparatus, product, or process disclosed, or represents that its use would not infringe privately owned rights. Reference herein to any specific commercial product, process, or service by trade name, trademark, manufacturer, or otherwise, does not necessarily constitute or imply its endorsement, recommendation, or favoring by the United States Government or any agency thereof. The views and opinions of authors expressed herein do not necessarily state or reflect those of the United States Government or any agency thereof.

\section{Availability}

This report is posted on the U.S. Department of Energy's Princeton Plasma Physics Laboratory Publications and Reports web site in Fiscal Year 2002. The home page for PPPL Reports and Publications is: http://www.pppl.gov/pub_report/

DOE and DOE Contractors can obtain copies of this report from:

U.S. Department of Energy

Office of Scientific and Technical Information

DOE Technical Information Services (DTIS)

P.O. Box 62

Oak Ridge, TN 37831

Telephone: (865) 576-8401

Fax: (865) 576-5728

Email: reports@adonis.osti.gov

This report is available to the general public from:

National Technical Information Service

U.S. Department of Commerce

5285 Port Royal Road

Springfield, VA 22161

Telephone: 1-800-553-6847 or

(703) 605-6000

Fax: (703) 321-8547

Internet: http://www.ntis.gov/ordering.htm 


\title{
Evaluation of Possible Nuclear Magnetic Resonance Diagnostic Techniques for Tokamak Experiments
}

\author{
S.J. Zweben, T. W. Kornack, D. Majeski, G. Schilling, C.H. Skinner, R. Wilson \\ Princeton Plasma Physics Laboratory, Princeton NJ 08543 \\ N. Kuzma \\ Princeton University, Princeton, NJ 08540
}

\begin{abstract}
Potential applications of nuclear magnetic resonance (NMR) diagnostic techniques to tokamak experiments are evaluated. NMR frequencies for hydrogen isotopes and low-Z nuclei in such experiments are in the frequency range $\approx 20-200 \mathrm{MHz}$, so existing RF antennas could be used to rotate the spin polarization and to make the NMR measurements. Our tentative conclusion is that such measurements are possible if highly spin polarized $\mathrm{H}$ or ${ }^{3} \mathrm{He}$ gas sources (which exist) are used to fuel these plasmas. In addition, NMR measurements of the surface layers of the first wall (without plasma) may also be possible, e.g. to evaluate the inventory of tritium inside the vessel.
\end{abstract}




\section{Introduction}

The use of nuclear magnetic resonance (NMR) for physical, chemical and biological studies is very well developed and highly successful [1-3]. Modern NMR spectrometers measure the resonances between an applied oscillating RF magnetic field and the nuclear spin states of a sample in a strong DC magnetic field. The NMR frequency and spin relaxation times vary with the local electronic and molecular environment, so NMR is used for quantitative chemical analysis of solids, liquids, and gases. Modern NMR devices can measure the structure of $\mu \mathrm{m}$-sized biological cells and even a single quantum dot on a $10 \mathrm{~nm}$ scale.

As far as we know there have been no attempts to use magnetic resonance to measure the properties of plasmas. However, there was a significant effort starting in 1982 to evaluate the properties of spin polarized nuclei in tokamak plasmas with the goal of increasing the fusion reaction rate [4-6]. Those papers calculated many of the relevant interactions between nuclear spins and the fusion plasmas which are of interest for plasma diagnostic applications. There was also an attempt to explain ion cyclotron emission in terms of spin-flip transitions [7].

The present paper examines some possible applications of NMR techniques to tokamak experiments. In general, NMR measurements on magnetized plasmas are difficult due to their low density and to the complexity of RF wave propagation in plasmas. However, our tentative conclusion is that such measurements are possible if highly spin polarized hydrogen or ${ }^{3} \mathrm{He}$ gas sources (which exist) are used to fuel these plasmas. In addition, NMR measurements of the surface layers of the first wall (without plasma) are also possible, e.g. to evaluate the inventory of tritium inside the vessel. 


\section{NMR in Plasmas}

\section{A. Background}

As illustrated in Table 1, many low-Z nuclei have non-zero nuclear spin and so can potentially be used for NMR measurements in tokamaks [1-3]. For example, the NMR frequency of a proton in a magnetic field of $B=2.35$ Tesla is $f_{N M R}=2 \mu B / h \approx 100$ $\mathrm{MHz}$, where $\mu$ is the nuclear magnetic moment of the proton. Normally only a very small fraction $\mathrm{F}_{\text {spin }} \approx \mu \mathrm{B} / \mathrm{kT}$ of the nuclei contribute to the NMR interaction in thermal equilibrium, e.g. for a proton with $\mu \approx 1.4 \times 10^{-26} \mathrm{~J} / \mathrm{T}, \mathrm{F}_{\text {spin }} \approx 8 \times 10^{-6}$ at this field at room temperature.

In a conventional pulsed NMR measurement, the sample is placed in a highly uniform magnetic field of typically $\mathrm{B} \leq 10 \mathrm{~T}$ and an RF driver coil outside the sample applies a pulse of oscillating magnetic field $\mathrm{B}_{1}$ transverse to $\mathrm{B}$. This $\mathrm{B}_{1}$ pulse rotates the nuclear spins from their equilibrium direction along $\mathrm{B}$ to a transient state with their net spins aligned transverse to B. After the driving RF field is turned off, these spins then begin to precess about $B$ at $f_{N M R}$ and produce a transverse magnetic field $B_{\perp}=\mu_{0} M_{\perp}$, where $\mathrm{M}_{\perp}=\mathrm{n} \mu$ is the transverse magnetization, $\mathrm{n}$ is the net density of nuclear spins, and $\mu_{0}$ $=4 \pi \times 10^{-7}$. The relaxation of these spins back to equilibrium, called the "free induction decay" (FID), can be measured with an RF antenna. The maximum output signal is the RF voltage induced in the pickup coil:

$$
\mathrm{V}(\text { volts })=\mathrm{N}_{\mathrm{c}} \mathrm{A}_{\mathrm{c}} \omega_{\mathrm{NMR}} \mathrm{B}_{\perp} \propto\left(\mu^{3} \mathrm{~B}^{2}\right)(\mathrm{n} / \mathrm{kT})
$$

where $\mathrm{N}$ is the number of turns in the pickup coil of area $\mathrm{A}\left(\mathrm{m}^{2}\right), \omega_{\mathrm{MMR}}$ is the $\mathrm{NMR}$ frequency $(\mathrm{rad} / \mathrm{sec})$, and $\mathrm{B}_{\perp}$ is the transverse magnetic field $(\mathrm{T})$. For example, for $1 \mathrm{~cm}^{3}$ of liquid water at room temperature with $\mathrm{B}=1$ Tesla, $\mathrm{F}_{\text {spin }} \approx 4 \times 10^{-6}, \mathrm{~B}_{\perp} \approx 3.4 \times 10^{-10} \mathrm{~T}$, and for coil with $\mathrm{N}=10$ turns at $\mathrm{A}=10^{-4} \mathrm{~m}^{2}$ per turn, the NMR signal voltage is $\mathrm{V} \approx 10^{-3}$ volt [8]. The relative signal level or "sensitivity" depends linearly on the sample density, linearly on the coil area, on the square of B, and on the cube of the nuclear moment (see Table 1). 


\section{B. Difficulties of NMR in Plasmas}

There are several properties of plasmas which make NMR measurements more difficult than those on solids or liquids, for example: (1) low density and high temperature, (2) highly non-uniform B field, (3) short ion confinement time and ion cyclotron orbital motion, and (4) complexity of RF wave propagation and RF "noise" near the NMR frequency. The following paragraphs discuss these difficulties for the case of ${ }^{3} \mathrm{He}$ nuclei Alcator C-Mod, where the maximum RF frequency of $80 \mathrm{MHz}$ corresponds to an NMR resonance at $\mathrm{B}=2.5 \mathrm{~T}$, and where typically $\mathrm{n}_{\mathrm{i}} \approx \mathrm{n}_{\mathrm{e}} \approx 2 \times 10^{14} \mathrm{~cm}^{-3}, \mathrm{~T}_{\mathrm{i}} \approx \mathrm{T}_{\mathrm{e}} \approx 2 \mathrm{keV}$.

If the nuclear spins of the plasma were in equilibrium at the room temperature, then the NMR signal for plasma protons at a given B would be smaller than that for water at room temperature by the density ratio (see Eq. 1), e.g. for an Ohmic Alcator C-Mod plasma roughly $\left.\approx\left[2 \times 10^{14} \mathrm{~cm}^{-3}\right) /\left(7 \times 10^{22} \mathrm{~cm}^{-3}\right)\right] \approx 3 \times 10^{-9}$. This ratio would be another $\approx 10^{5}$ times smaller if the plasma spins were at the plasma temperature. However, it is now possible to produce nearly $100 \%$ polarized hydrogen [9] or ${ }^{3} \mathrm{He}$ [10] gas which could be used to fuel the plasma. Assuming for the moment that the plasma retains this polarization (see below), the resulting signal for the plasma NMR becomes larger by $\left(1 / \mathrm{F}_{\text {spin }}\right)$, where $\mathrm{F}_{\text {spin }} \approx 8 \times 10^{-6}$ for protons at $\mathrm{B}=2.35 \mathrm{~T}$ at room temperature. Thus the NMR signal from a fully polarized proton plasma would be $\approx 3 \times 10^{-9} / 8 \times 10^{-6} \approx 4 \times 10^{-4}$ times that from liquid water, i.e. a plasma of area $\approx 1 \mathrm{~m}^{2}$ would have an NMR signal comparable to $\approx 1 \mathrm{~cm}^{3}$ of water (which was measurable in the 1940s).

However, the highly non-uniform B field in a toroidal plasma presents a major difficulty, since the NMR frequency of all species will vary as $1 / R$ with respect to the plasma major radius $\mathrm{R}$. Although this does allow spatial localization during the $\mathrm{B}_{1}$ "drive" similar to that used in Magnetic Resonance Imaging (MRI), the relatively large $\Delta \mathrm{B}$ over which these driven spins resonate will cause them to decohere rapidly during the FID. For example, if a $B_{1}$ pulse is applied with $\Delta \mathrm{f} / \mathrm{f} \approx 1 \%$ (i.e. $\Delta \mathrm{f} \approx 0.8 \mathrm{MHz}$ with $\tau_{\mathrm{RF}} \approx 1$ $\mu \mathrm{sec})$, then the NMR resonance would occur over a width $\sigma_{\Delta \mathrm{B}} \approx(\Delta \mathrm{B} / \mathrm{B}) \omega_{\mathrm{NMR}} \approx 1 \mathrm{MHz}$, resulting in $\mathrm{a} \approx 1 \mu \mathrm{sec}$ decay time for the FID. This broad spectrum decreases the signal/noise with respect to conventional NMR spectroscopy, which is done in a highly 
uniform B field (the applied gradient in MRI is turned off during the FID).

Furthermore, the high speed of plasma ions also cause broadening of the NMR resonance in plasmas. For example, if the NMR resonance region has a radial width of $\Delta \mathrm{B} / \mathrm{B} \approx 1 \%$, e.g. $\Delta \mathrm{R} \approx 0.7 \mathrm{~cm}$ in Alcator $\mathrm{C}-\mathrm{Mod}$, the ions would move out of the resonance region due to their poloidal motion with a frequency $\sigma_{\mathrm{pol}} \approx\left(\mathrm{v}_{\mathrm{i}} / \Delta \mathrm{R}\right)\left(\mathrm{B}_{\mathrm{pol}} / \mathrm{B}\right) \approx 1$ $\mathrm{MHz}$ in Alcator C-Mod. Other broadening effects due to the finite ion gyroradius, the ion Doppler shift, and ion radial diffusion should be small compared to $\sigma_{\mathrm{pol}}$.

Finally, another major difficulty for plasma NMR is the complexity of RF wave propagation into and out of the plasma. In general, the relationship between the NMR frequency and the ion cyclotron frequency is:

$$
\mathrm{f}_{\mathrm{NMR}} / \mathrm{f}_{\mathrm{ci}}=(1 / 2)\left(\mu / \mu_{\mathrm{N}}\right)\left(\mathrm{M}_{\mathrm{i}} / \mathrm{M}_{\mathrm{p}}\right)(1 / \mathrm{Z})
$$

where $\mu$ is the nuclear moment, $\mu_{\mathrm{N}}$ is the nuclear magneton, and the ion has a charge $\mathrm{Z}$ and a mass $M_{i}$ with respect to the proton mass $M_{p}$. For most cases $f_{N M R}>f_{c i}$ (Table 1), so $\mathrm{RF}$ waves near $\mathrm{f}_{\mathrm{NMR}}$ should propagate relatively well in tokamaks. However, there also spontaneous RF emission from the plasma which will be a "background" for any NMR measurements. These issues are discussed in more detail in Sec. III.B and III.C.

\section{Nuclear Spin Relaxation Processes in Plasma}

In their analysis of the possibility of spin-polarized fusion plasmas, Kulsrud et al [4-6] calculated various relaxation rates for the polarization of deuterium and tritium nuclei in the core of a magnetic fusion plasma with $B=5 \mathrm{~T}, \mathrm{n}=10^{14} \mathrm{~cm}^{-3}$ and $\mathrm{T}=10 \mathrm{keV}$. They concluded that the cross-section for depolarization through binary collisions is small compared to the fusion reaction cross section, so collisional spin relaxation should be negligible for magnetic fusion plasmas of interest. They also showed that ionization, charge exchange, and recombination will not significantly change the spin state of hydrogen isotopes in this strong magnetic field; thus the net polarization of the plasma (in the absence of other depolarization mechanisms) should retain the polarization of the 
neutrals used to fuel the plasma. They also showed that ion thermal and ion cyclotron motion in an sheared inhomogeneous magnetic field should cause a negligible depolarization, even with collisions. However, when a polarized nucleus contacts the wall surface it can depolarize rapidly [11]; therefore the polarization will tend to decay on a particle confinement time. Thus without direct fueling by polarized nuclei, the plasma spins would most likely retain the net polarization of their source, which could be the first wall (for recycling species), or the fueling source from gas puffing, pellets, or NBI.

The only other source of nuclear spin depolarization identified by Kulsrud et al is that due to internal plasma magnetic fluctuations in the frequency range $\approx(1 / 2-2) \omega_{\mathrm{NMR}}$. $\mathrm{A}$ rough estimate for the amount of depolarization rate $\sigma_{\delta \mathrm{B}}$ for tritons due to a left circularly polarized wave with a mean-square amplitude $\delta B_{\perp}$ perpendicular to $B$ was [5]: $\sigma_{\delta B} \approx 1 / 8$ $\left(\delta \mathrm{B}_{\perp} / \mathrm{B}\right)^{2} \omega_{\mathrm{NMR}}$. Thus for $\mathrm{B}=5 \mathrm{~T}$ and an assumed $\delta \mathrm{B}_{\perp} \approx 10^{-4} \mathrm{~T}$, tritons would depolarization within $\approx 75 \mathrm{msec}$, which is short compared with the triton particle confinement time. Thus if there were no other faster decorrelation mechanisms, a measurement of the decorrelation rate of nuclear spins could potentially be used as a diagnostic for internal magnetic field fluctuations, assuming the gas fueling rate is faster than the internal decorrelation rate.

III. NMR measurements on a tokamak

A general scenario for NMR measurements in a tokamak is illustrated in Fig. 1. The plasma would be started as usual but would then be fueled with a highly spin polarized gas. Just after fueling is completed, a series of $\mathrm{RF} \mathrm{B}_{1}$ pulses of various amplitudes would be applied through an RF antenna, and after each pulse the FID would be measured by the same (or another) RF antenna. This pulse sequence and FID measurement would be repeated later on until the NMR response has diminished to nearly zero.

\section{A. Estimate of NMR signal}

For clarity, we will initially assume that the tokamak is Alcator C-Mod and that the polarized fuel is ${ }^{3} \mathrm{He}$. The existing RF system has antennas tuned to a maximum 
frequency of $80 \mathrm{MHz}$, which corresponds to a ${ }^{3} \mathrm{He}$ NMR resonance at $\mathrm{B} \approx 2.5 \mathrm{~T}$. Assuming for the moment that all ${ }^{3} \mathrm{He}$ ions in the plasma have their spins rotated $90^{\circ}$ by the RF pulse to produce the maximum transverse magnetic field, the NMR signal can be estimated as follows (see Sec. II.A): $M_{\perp}=n \mu \approx\left(2 \times 10^{20} \mathrm{~m}^{-3}\right)\left(2.1 \cdot 5 \times 10^{-27} \mathrm{~J} / \mathrm{T}\right)=2.1 \times 10^{-6}$ amp-turn $/ \mathrm{m}$, thus $B_{\perp}=\mu_{0} M_{\perp} \approx\left(4 \pi \times 10^{-7}\right)\left(2.1 \times 10^{-6}\right.$ amp-turn $\left./ \mathrm{m}\right)=2.6 \times 10^{-12}$ Tesla, and so $\mathrm{V}_{\mathrm{NMR}}=\mathrm{NA} \omega_{\mathrm{NMR}} \mathrm{B}_{\perp} \approx\left(0.3 \mathrm{~m}^{2}\right)\left(5 \times 10^{8} \mathrm{rad} / \mathrm{sec}\right)\left(2.6 \times 10^{-12} \mathrm{~T}\right) \approx 4 \times 10^{-4}$ volts.

However, in toroidal geometry only a small fraction of the plasma ions would be resonant with a given $\mathrm{RF}$ pulse due to the (1/R) toroidal field (as usual for ICRH heating). Assuming an RF driver pulse of duration $\tau_{\mathrm{RF}} \approx 1 \mu$ sec (i.e. with a $1 \mathrm{MHz}$ bandwidth at $80 \mathrm{MHz}$ ), only the ${ }^{3} \mathrm{He}$ nuclei within $\Delta \mathrm{B} / \mathrm{B} \approx \Delta \mathrm{R} / \mathrm{R} \approx 1 \%$ on a vertical cylinder at $\mathrm{R}=\mathrm{R}_{0}$ would be resonant. This $\Delta \mathrm{R} \approx 0.5 \mathrm{~cm}$ wide region would contain $\approx 2 \%$ of the plasma ions. Since the NMR signal is proportional to the number of resonant ions, the NMR voltage at the antenna would be $\mathrm{V}_{\mathrm{NMR}} \approx(0.02)\left(4 \times 10^{-4}\right) \approx 10^{-5}$ volts. The spectrum of the FID after the RF pulse would have a bandwidth of $\approx 1 \mathrm{MHz}$ due to the finite $\Delta \mathrm{B}$ and poloidal ion motion, as discussed in Sec. II.B.

Therefore it seems possible to detect the NMR response of a fully polarized tokamak plasma. The detected signal could be calibrated by using a known pressure of polarized ${ }^{3} \mathrm{He}$ gas in the chamber (without a plasma). The "tipping angle" of the rotated spins could be determined by finding the maximum response vs. the RF pulse amplitude, and various pulse sequences could be tried. Fueling with polarized ${ }^{3} \mathrm{He}$ gas should be feasible as long as a small magnetic field is applied to the gas bottle and tubing the interactions with the tube surface are minimized by a reasonably fast flow rate.

\section{B. Estimate of noise and background levels}

The main background for conventional NMR measurements is the thermal noise in the RF detector, $V_{\text {th }}=\left(4 \mathrm{kT}_{\text {det }} \Omega \Delta \mathrm{f}\right)^{1 / 2}$, where $\mathrm{k}$ is Boltzman's constant, $\mathrm{T}_{\text {det }}$ is the detector temperature $\left({ }^{\circ} \mathrm{K}\right), \Omega$ is the detector resistance $(\mathrm{Ohms})$, and $\Delta \mathrm{f}$ is the signal bandwidth $(\mathrm{Hz})$. Thus for an RF antenna at room temperature with a resistance of $\Omega=50$ ohms, the thermal noise for a bandwidth of $1 \mathrm{MHz}$ is $\mathrm{V}_{\text {th }} \approx 10^{-6}$ volts, which is about 0.1 
times the expected NMR signal. This noise level is unusually large for NMR measurements due to the large bandwidth of the plasma NMR signal compared with that of liquids or solids.

Another source of noise would be the "ion cyclotron emission" (ICE) normally seen as RF emission from tokamaks at harmonics of $\omega_{\text {ci }}[7,12]$. This noise level would need to be measured on each machine, but for JET it was typically $-80 \mathrm{dBm}$ in the absence of fusion products (as would be the case for ${ }^{3} \mathrm{He}$ in Alcator). For an antenna of $\mathrm{R}=50 \Omega$ this is would produce a noise voltage of $\mathrm{V}_{\text {ICE }} \approx 2 \times 10^{-5}$ volts, which about twice the expected NMR signal. Therefore ICE emission could be a significant noise source.

Another background in these plasma NMR measurements would be the low-Z nuclei on the first wall surface within the range of radii at the resonant B field. Although the number of ${ }^{3} \mathrm{He}$ nuclei on the surface would be negligible, there would be an NMR resonance with $\mathrm{H}$ nuclei at a radius 0.76 times that at which the ${ }^{3} \mathrm{He}$ resonate (see Table 1 ). Assuming $\approx 10^{4}$ monolayers of $\mathrm{H}$ on the first wall, the surface density would be $\approx$ $10^{20}$ atoms $/ \mathrm{cm}^{2}$, of which only a small fraction $\mathrm{F}_{\text {spin }} \approx 10^{-5}$ would be polarized at room temperature. Thus the number of nuclei per $\mathrm{cm}^{2}$ of wall surface would be $\approx 10^{14} \mathrm{~cm}^{-2}$, whereas the density of resonant ${ }^{3} \mathrm{He}$ nuclei in the plasma would be $\approx\left(2 \times 10^{14} \mathrm{~cm}^{-3}\right)(50 \mathrm{~cm})$ $\approx 10^{16}$ atoms $/ \mathrm{cm}^{2}$, i.e. the plasma signal should be $\approx 100$ times this background. Note that the D resonance would be outside the machine since its NMR frequency is so low (see Table 1).

\section{Propagation of RF and NMR signal}

It is well known that RF waves can propagate from antennas to the plasma center at frequencies well above the ion cyclotron frequency $\omega_{\text {ci, }}$, so these waves should be able to penetrate to the plasma center to rotate the nuclear spin polarization. However, these fast Alfven waves normally have only a small component of polarization in the direction perpendicular to $\mathrm{B}$ (which is needed to rotate the nuclear polarization of ${ }^{3} \mathrm{He}$ ), although this would probably be sufficient given the large RF power available in existing tokamak experiments $(\approx 1-10 \mathrm{MW})$. Due to the complexity of this propagation the magnitude of 
these waves can not be accurately predicted, therefore the RF pulse amplitude would have to be varied in order to calibrate the angle of spin "tipping" (see Fig. 1). Similarly, the small fluctuating magnetic fields caused by the nuclear precession during the FID inside the plasma center should be able to propagate to the RF antennas to be detected. This would most likely occur through the conversion to fast Alfven waves near the center, although the efficiency of this process is not well known.

\section{Magnetic field fluctuation measurement}

Perhaps the most interesting NMR application to a tokamak would be a measurement of the internal magnetic field fluctuations. As noted in Sec. II.C, transverse magnetic field fluctuations $\delta \mathrm{B}_{\perp}$ in the NMR frequency range are the most likely source of nuclear depolarization inside a hot plasma; thus if the decay time of the spin polarization can be measured, then this might be used to infer $\delta \mathrm{B}_{\perp}$. However, since the nuclei in a tokamak circulate rapidly around a flux surface, this would measure the average $\delta \mathrm{B}_{\perp}$ over the ${ }^{3} \mathrm{He}$ ion orbits, rather than its local value at the NMR resonance.

In principal, this spin decorrelation time could be measured as a function of minor radius (by varying the applied RF frequency or bandwidth), or as a function of the fluctuation frequency at a given radius using different species, e.g. D for low and $\mathrm{T}$ for high frequency waves. The frequency spectrum of this $\delta \mathrm{B}_{\perp}$ measurement might also be "fine-tuned" by varying the parallel wavenumber of the RF driving waves, thus varying the effective NMR frequency [5]. The response of the net polarization to a known $\delta B_{\perp}$ could be calibrated by introducing known RF waves via the external antennas; in fact, the NMR measurement process itself should produce a few-\% depolarization per RF pulse, equal to the fraction of resonant spins in the plasma.

However, it should be noted that a plasma magnetic fluctuation level of $\approx 10^{-4} \mathrm{~T}$ would be $\approx 10^{8}$ times larger than the fluctuating magnetic field expected from the nuclear spin precession (see Sec. III.A). Thus the fluctuating plasma magnetic fields could

potentially be a large source of background in these experiments. It is somewhat surprising that these plasma fluctuations are not already detected at the RF antennas as 
ICE; perhaps this is due to their relatively short coherence length compared to the RF wavelength .

\section{E. Species measurement}

One of the main uses of NMR in solids and liquids is to identify the chemical composition of samples via the spectrum of the NMR response. It would be useful if NMR could be used to measure the H/D/T species mix and low-Z impurity content of the

plasma. However, the large variation of magnetic field across the plasma causes the NMR resonances of several species to overlap, e.g. at a given NMR frequency the $H$ resonance would be at a radius 1.06 larger than the $\mathrm{T}$ resonance. Also, each of these species would probably need to be highly polarized to be measurable. Thus it does not seem feasible to use NMR to determine the species mix inside the plasma in a tokamak (however, this technique might be usable in a linear plasma device with a uniform B).

\section{F. Surface measurements (without plasma)}

NMR techniques could potentially be used to measure the surface composition of the first wall in the absence of plasma. These surface layers are typically composed of $\approx 10-100 \mu \mathrm{m}$ thick coatings of low-Z elements, several of which have NMR responses (see Table 1), e.g. tritium, $\mathrm{Li}, \mathrm{Be}$ and B. This measurement could use the near fields from the normal RF antennas, or specially designed RF drive/pickup coils positioned using a remotely controlled arm inside the vessel. These fields should fully penetrate these coatings since the RF skin depths at $100 \mathrm{MHz}$ are $\approx 50 \mu \mathrm{m}$ in stainless steel and 2 $\mathrm{mm}$ in carbon. The NMR signal levels would be $\approx 100$ times lower than for a fully polarized plasma (see Sec. III.B); however, the noise from ICE would be absent, and the NMR pulse could be repeated many times during a TF pulse to increase the S/N level.

A special application of this measurement could be used to evaluate the tritium inventory inside a fusion device like JET or ITER. Tritium has the largest magnetic moment of any nucleus, and tritium levels as low as $0.1 \mathrm{mCi}$ have been measured using NMR [13]. This technique could potentially scan over $\mathrm{R}$ by varying $\mathrm{B}$, and could be calibrated using known tritium gas concentrations in the vessel. It might also be possible 
to use the nearly constant vertical field instead of the toroidal field for such measurements. Wall samples could also be removed from the chamber for analysis in a conventional NMR spectrometer.

Acknowledgments: We thank for their helpful comments W. Arunasalam, W. Happer, D.W. Johnson, and R. Kulsrud. This work was performed under DOE contract \# DEAC02-76CHO3073. 
Table 1: Nuclear Magnetic Resonance Parameters

\begin{tabular}{lllll} 
Particle & Spin & $\mathrm{f}_{\underline{\underline{M M R}}}(\mathrm{MHz})^{*}$ & $\mathrm{f}_{\underline{\mathrm{MMR}}} \underline{\mathrm{f}_{\mathrm{ci}} * *}$ & Sensitivity $* * *$ \\
\hline Hydrogen & $1 / 2$ & 100 & 2.8 & 1.00 \\
Deuterium & 1 & 15.35 & 0.86 & 0.009 \\
Tritium & $1 / 2$ & 106.7 & 9.0 & 1.2 \\
${ }^{3} \mathrm{He}$ & $1 / 2$ & 76.2 & 3.2 & 0.44 \\
${ }^{6} \mathrm{Li}$ & 1 & 14.7 & 0.82 & 0.009 \\
${ }^{7} \mathrm{Li}$ & $3 / 2$ & 38.9 & 2.5 & 0.29 \\
${ }^{9} \mathrm{Be}$ & $3 / 2$ & 14.1 & 0.89 & 0.014 \\
${ }^{10} \mathrm{~B}$ & 3 & 10.7 & 0.6 & 0.02 \\
${ }^{11} \mathrm{~B}$ & $3 / 2$ & 32.1 & 2 & 0.17 \\
${ }^{13} \mathrm{C}$ & $1 / 2$ & 25.1 & 1.5 & 0.016
\end{tabular}

* at B=2.3488 T, from Abraham et al, Introduction to NMR Spectroscopy, 1988

** for fully ionized nuclei, equal to $\mathrm{f}_{\mathrm{NMR}} / \mathrm{f}_{\mathrm{ci}}=(1 / 2)\left(\mu / \mu_{\mathrm{N}}\right)\left(\mathrm{M}_{\mathrm{i}} / \mathrm{M}_{\mathrm{p}}\right)(1 / \mathrm{Z})$

*** relative to protons at constant $\mathrm{B}$ for equal number of nuclei 


\section{References:}

[1] R.J. Abraham, J. Fisher, and P. Loftus, Introduction to NMR Spectrosccopy, John Wiley and Sons, Chichester UK 1988

[2] F.A. Bovey et al, Nuclear Magnetic Resonance Spectroscopy $2^{\text {nd }}$ Edition, Academic Press, San Diego 1988

[3] P.J. Hore, Nuclear Magnetic Resonance, Oxford University Press, Oxford UK 1995

[4] R.M. Kulsrud, H.P. Furth, E.J. Valeo, M. Goldhaber, Phys. Rev. Lett.. 49, 1248 (1982)

[5] R.M. Kulsrud, E.J. Valeo and S.C. Cowley, Nucl. Fusion 26, 1443 (1986)

[6] S.C. Cowley, R.M. Kulsrud, and E. Valeo, Phys. Fluids 29, 430 (1986)

[7] W. Arunasalam, G.J. Greeene, K.M. Young, Nucl. Fusion 34, 927 (1994)

[8] F. Bloch, Phys. Rev. 70, 460 (1946)

[9] R.W. Cline, T.J. Greytak, D.Kleppner,Phys. Rev. Lett. 47, 1195 (1981)

[10] T.E. Chupp, M.E. Wagshul, K.P. Coulter, A.B. McDonald, and W. Happer, Phys. Rev. C 36, 2244 (1987)

[11] H.S. Greenside, R.V. Budny, and D.E. Post, J. Vac. Sci. Technol. A, 2619 (1984)

[12] G.A. Cottrell and R.O. Dendy, Phys. Rev. Lett. 60, 33 (1988)

[13] E.A. Evans et al, Handbook of Tritium NMR Spectroscopy, WileyInterscience, Chichester UK 1985 
Figure 1: Scenario for NMR measurements in a tokamak plasma.

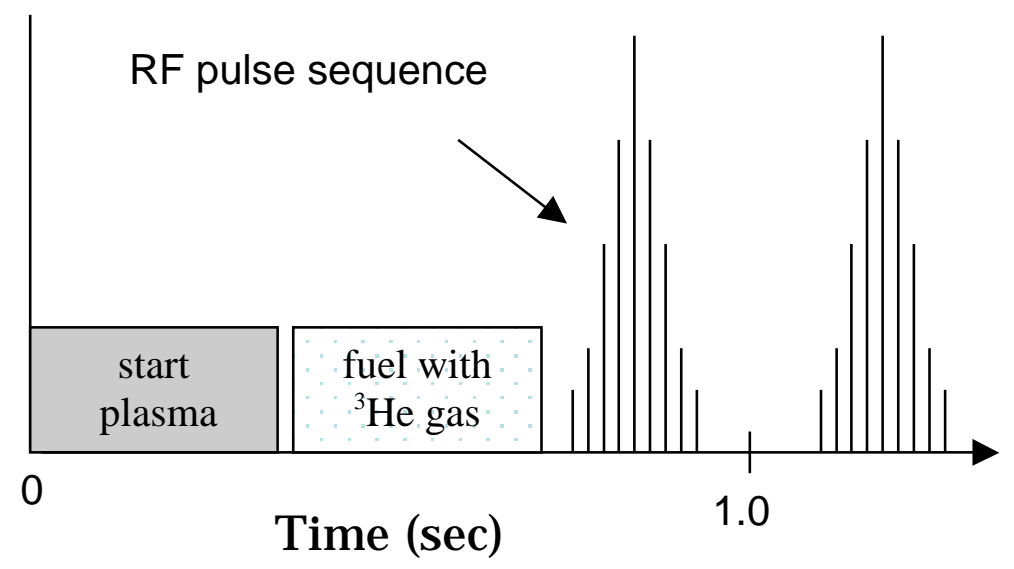




\section{External Distribution}

Plasma Research Laboratory, Australian National University, Australia

Professor I.R. J ones, Flinders University, Australia

Professor J oão Canalle, Instituto de Fisica DEQ/IF - UERJ , Brazil

Mr. Gerson O. Ludwig, Instituto Nacional de Pesquisas, Brazil

Dr. P.H. Sakanaka, Instituto Fisica, Brazil

The Librarian, Culham Laboratory, England

Library, R61, Rutherford Appleton Laboratory, England

Mrs. S.A. Hutchinson, JET Library, England

Professor M.N. Bussac, Ecole Polytechnique, France

Librarian, Max-Planck-Institut für Plasmaphysik, Germany

J olan Moldvai, Reports Library, MTA KFKI-ATKI, Hungary

Dr. P. Kaw, Institute for Plasma Research, India

Ms. P.J . Pathak, Librarian, Insitute for Plasma Research, India

Ms. Clelia De Palo, Associazione EURATOM-ENEA, I taly

Dr. G. Grosso, Instituto di Fisica del Plasma, Italy

Librarian, Naka Fusion Research Establishment, J AERI, J apan

Library, Plasma Physics Laboratory, Kyoto University, J apan

Research Information Center, National Institute for Fusion Science, J apan

Dr. O. Mitarai, Kyushu Tokai University, J apan

Library, Academia Sinica, Institute of Plasma Physics, People's Republic of China

Shih-Tung Tsai, Institute of Physics, Chinese Academy of Sciences, People's Republic of China

Dr. S. Mirnov, TRINITI, Troitsk, Russian Federation, Russia

Dr. V.S. Strelkov, Kurchatov Institute, Russian Federation, Russia

Professor Peter Lukac, Katedra Fyziky Plazmy MFF UK, Mlynska dolina F-2, Komenskeho Univerzita, SK-842 15 Bratislava, Slovakia

Dr. G.S. Lee, Korea Basic Science Institute, South Korea

Mr. Dennis Bruggink, Fusion Library, University of Wisconsin, USA

Institute for Plasma Research, University of Maryland, USA

Librarian, Fusion Energy Division, Oak Ridge National Laboratory, USA

Librarian, Institute of Fusion Studies, University of Texas, USA

Librarian, Magnetic Fusion Program, Lawrence Livermore National Laboratory, USA

Library, General Atomics, USA

Plasma Physics Group, Fusion Energy Research Program, University of California at San Diego, USA

Plasma Physics Library, Columbia University, USA

Alkesh Punjabi, Center for Fusion Research and Training, Hampton University, USA

Dr. W.M. Stacey, Fusion Research Center, Georgia Institute of Technology, USA

Dr. J ohn Willis, U.S. Department of Energy, Office of Fusion Energy Sciences, USA

Mr. Paul H. Wright, Indianapolis, Indiana, USA 
The Princeton Plasma Physics Laboratory is operated by Princeton University under contract with the U.S. Department of Energy.

\author{
Information Services \\ Princeton Plasma Physics Laboratory \\ P.O. Box 451 \\ Princeton, NJ 08543
}

Phone: 609-243-2750

Fax: 609-243-2751

e-mail: pppl_info@pppl.gov

Internet Address: http://www.pppl.gov 